\title{
Arthrospira (Spirulina) platensis Attenuates Dextran Sulfate Sodium-induced Colitis in Mice by Suppressing Key Pro-inflammatory Cytokines
}

\author{
Francisca Adilfa de Oliveira Garcia ${ }^{1,4^{*}}$, Helioswilton Sales-Campos ${ }^{2,5^{*}}$, Violet G. Yuen ${ }^{1}$, \\ Juliana Reis Machado ${ }^{2}$, Glauce Socorro de Barros Viana ${ }^{3,4}$, Carlo José Freire Oliveira ${ }^{\prime}$ and \\ John H. McNeill ${ }^{1}$ \\ Faculty of Pharmaceutical Sciences, The University of British Columbia (UBC), Vancouver ${ }^{1}$, British Columbia, Canada; \\ Departamento de Microbiologia, Imunologia e Parasitologia, Universidade Federal do Triangulo Mineiro (UFTM) ${ }^{2}$, \\ Minas Gerais, Brazil; Departamento de Farmacologia, Universidade Federal do Ceará (UFC) ${ }^{3}$, Fortaleza, CE, Brazil; \\ Departamento de Fisiologia, Faculdade de Medicina Estacio de Juazeiro do Norte (ESTACIO) ${ }^{4}$, Juazeiro do Norte, CE, \\ Brazil; Instituto de Patologia Tropical e Saúde Pública, Universidade Federal de Goiás ${ }^{5}$, Goiânia, GO, Brazil
}

Background/Aims: Therapies aimed at modulating cytokines have been used to treat inflammatory illnesses, such as inflammatory bowel disease. On the other hand, patients may become intolerant, refractory, or present with several side effects. Arthrospira (Spirulina) platensis (SPI) is a blue-green microalga with bioactive molecules that have been evaluated to treat inflammatory diseases. On the other hand, few studies have examined their effects on the production of specific cytokines and the intestinal architecture in dextran sulfate sodium (DSS)-induced colitis. Therefore, this study examined the effects of a treatment using SPI in a murine model of intestinal inflammation.

Methods: All mice (C57BL/6 male) were evaluated daily for their food and water intake, bodyweight variations, and clinical signs of disease. Colon inflammation was induced by exposure to DSS for 6 consecutive days. SPI was given orally at 50,100 , and 250 $\mathrm{mg} / \mathrm{kg} /$ day. ELISA was performed to assess the production of cytokines. Myeloperoxidase and nitric oxide were also investigated. The level of microscopic damage was assessed by staining colon sections with hematoxylin and eosin.

Results: SPI attenuated the DSS-induced inflammation, with improvements in the clinical signs and a decrease in the production of inflammatory cytokines, such as tumor necrosis factor- $\alpha$ and interferon- $\gamma$. In addition, particularly at $250 \mathrm{mg} / \mathrm{kg}, \mathrm{SPI}$ attenuated the severity of colitis by modulating the level of mucosal and submucosal cell infiltration, which preserved the epithelial barrier.

Conclusions: SPI may be an alternative source of bioactive molecules with immunomodulatory properties, and has great potential to be used in the treatment of inflammatory diseases. (Korean J Gastroenterol 2020;76:150-158)

Key Words: Spirulina; Inflammatory bowel diseases; Cytokines; Immunomodulation; Inflammation

\section{INTRODUCTION}

Ulcerative colitis (UC) and Crohn's disease (CD) are the ma- jor entities of inflammatory bowel disease. These disorders are immune-mediated diseases characterized by an imbalance between the regulatory and effector immune re-

Received May 8, 2020. Revised June 17, 2020. Accepted June 20, 2020.

(C) This is an open access article distributed under the terms of the Creative Commons Attribution Non-Commercial License (http://creativecommons.org/licenses/ by-nc/4.0) which permits unrestricted non-commercial use, distribution, and reproduction in any medium, provided the original work is properly cited. Copyright (C) 2020. Korean Society of Gastroenterology.

Correspondence to: Helioswilton Sales-Campos, Instituto de Patologia Tropical e Saúde Pública, Universidade Federal de Goiás, Rua 235 S/N Setor Leste Universitário, Goiánia, Goiás 746050-050, Brazil. Tel: +55-62-3209-6109, Fax: +55-62-3209-6363, E-mail address: tonsales@ufg.br, ORCID: https://orcid.org/ 0000-0003-3252-2834

Financial support: Fundação de Amparo à Pesquisa do Estado de Minas Gerais (FAPEMIG), Coordenação de Aperfeiçoamento de Pessoal de Nível Superior (CAPES), and Conselho Nacional de Desenvolvimento Científico e Tecnológico (CNPq). Conflict of interest: None.

*These authors contributed equally to this work. 
sponses in the gut where immune cells respond to autoantigens, thereby triggering chronic inflammation. ${ }^{1}$ IBD is characterized by the production of pro-inflammatory cytokines, such as interferon (IFN)- $\gamma$, tumor necrosis factor (TNF)$\alpha$, interleukin (IL)-6, and IL-17. ${ }^{2}$ Current therapies have limitations, despite reducing the production of inflammatory cytokines and influencing the disease outcomes. In addition, patients may become refractory or intolerant and presenting with several side effects. ${ }^{3}$ These constrictions reinforce the necessity to develop new therapeutic approaches.

Arthrospira (Spirulina) platensis (SPI), commonly known as the nutraceutical spirulina, is a planktonic, filamentous cyanobacterium largely used as a source of bioactive molecules, such as amino acids, vitamins, carotenoids, and minerals. SPI is used frequently for nutrient supplementation in humans without any significant side effects. ${ }^{4,5}$ An extract of SPI has also been used to treat inflammatory disturbances in experimental models of arthritis, ${ }^{6-8}$ non-alcoholic steatohepatitis, ${ }^{9}$ and in patients with allergic rhinitis. ${ }^{10}$ The beneficial role of SPI has been addressed in experimental models of 2, 4, 6-trinitrobenzene sulfonic acid (TNBS) ${ }^{11}$ and acetic acid-induced intestinal inflammation ${ }^{12}$, which have similarities to the pathophysiology observed in $\mathrm{CD}^{13}$ and $\mathrm{UC},{ }^{14}$ respectively. More recently, the beneficial effects of SPI in dextran sulfate sodium (DSS)-induced colitis have been examined in rats. ${ }^{15}$

The effects of SPI extracts have never been explored in DSS-induced intestinal inflammation in mice. The DSS model is one of the most used approaches to study inflammatory bowel disease (IBD) and is believed to resemble the features of flares in $\mathrm{UC}^{13}$ and $\mathrm{CD}$ (mainly transmural inflammation). ${ }^{16}$ Therefore, this study examined the effects of an SPI extract on the clinical outcomes, colon architecture, and production of the innate and adaptive immune mediators in a murine model of DSS-induced intestinal inflammation.

\section{SUBJECTS AND METHODS}

\section{Cultivation and collection of Arthrospira (Spirulina) platensis}

The cultivation of SPI requires intense sunlight, high temperatures and low rainfall, nutrients, and a pH between 9 and 10. Initially, the inocula received from Antenna (Geneve, Switzerland) were cultivated in the authors' laboratory at the Federal University of Ceara, Brazil, utilizing Zarrouk media.
After filtration, the material was weighed to determine the wet biomass and submitted to desiccation for 5 hours $\left(50^{\circ} \mathrm{C}\right)$. The dried material was weighed to determine the dried biomass and the production per square meter ( 8 to $10 \mathrm{~g} / \mathrm{day} / \mathrm{m}^{2}$ ).

\section{Animal experimentation}

C57BL/6 male mice aged 6-8 weeks, weighing 20-25 g, were divided randomly into five groups, with six mice/groups, as follows: healthy-mice not exposed to DSS and treated with saline only; DSS+Vei-mice exposed to 3\% DSS and treated with saline by gavage; DSS+50, 100 or $250 \mathrm{mg} / \mathrm{kg} /$ day-mice exposed to DSS $3 \%$ and treated by gavage with different concentrations of SPI. Colitis was induced by 3\% DSS (MP Biomedicals, Illkirch, France, Molecular weight: 36,000-50,000 $\mathrm{kDa}$ ) added continuously to the drinking water for six consecutive days. Saline and SPI were given orally by gavage for five days. After this period, the mice were euthanized with an intraperitoneal injection of 2, 2, 2-tribromoethanol followed by a cervical dislocation, and the colon samples were collected for immunological and histological assays. The results are representative of two independent experiments. The extract was obtained by macerating SPI dried biomass with saline to obtain the target concentrations.

All animals were housed in specific pathogen-free and standard-controlled environmental conditions at a constant temperature $\left(25^{\circ} \mathrm{C}\right)$ on a 12 hours light/dark cycle and provided with food and water ad libitum in the animal housing facility of the Federal University of Triângulo Mineiro, Brazil. The Institutional Animal Care and Use Committee of Federal University of Triângulo Mineiro approved this study under protocol 388, and all procedures were performed in accordance with the international ethics guidelines. All mice were monitored for their general wellbeing before, during, and after the experiments, as described previously. ${ }^{17}$

\section{Euthanasia and sample collection}

The mice were euthanized on day six, and the colon was removed for further analysis. The colon length was evaluated immediately, and the samples were divided into two smaller sections: 1) immersed into PBS/10\% formaldehyde for paraffin embedding; and 2) immersed in a solution containing phosphate-buffered saline (PBS), 2\% Nonidet P-40 and protease inhibitors (Complete ${ }^{\circledR}$, Roche Pharmaceuticals, Mannheim, Germany) for myeloperoxidase (MPO), nitric oxide (NO) and 
cytokine/chemokine quantification. The dry weights of all sections were determined before storage.

\section{Clinical assessment of mice with DSS-induced colitis}

All mice were evaluated daily for their food and water intake, bodyweight variation, and clinical signs of disease. The clinical, overall clinical, post mortem, and accumulated scores were assessed to obtain a disease score for every mouse, as described previously. ${ }^{18}$ Briefly, each sign presented by the animals (wet anus, bleeding stools, stools consistency, piloerection, and hypoactivity) corresponded to one point, and the sum of points for each mouse was defined as the overall clinical score. For weight variation, mice that lost 5 to $10 \%$ of their body weight in 24 hours received one point. Weight losses higher than $10 \%$ received two points. The percentage of weight variation was calculated as follows: the weight of each mouse was determined at the beginning of the study and was considered to be $100 \%$. The weight variation was calculated for each animal during the experimental period based on the daily weight gain or loss and compared to their weight on day 0 . The post mortem score was calculated based on the macroscopical alterations of the colon (consistency of stool and bleeding). One point was attributed to each alteration if these alterations were not diffused. Regarding the diffused alterations, two points were attributed to each alteration observed. The accumulated score was calculated based on the clinical score on the day of euthanasia and the macroscopic score. The sum of the signs or macroscopic disturbances for each mouse was represented graphically as a bar chart.

\section{Intestinal homogenate preparation}

Colon sections stored in PBS containing the protease inhibitors were kept at $4{ }^{\circ} \mathrm{C}$ and disrupted mechanically using TissueRuptor II (Qiagen, Germantown, MD, USA) until visual homogenization was achieved. The samples were then centrifuged at $1,000 \mathrm{xg}$, and the supernatants were stored at $-80^{\circ} \mathrm{C}$ until the MPO, NO, and cytokine quantification.

\section{MPO and NO analysis}

The activity of MPO was determined as previously described. ${ }^{19}$ Briefly, a tetramethylbenzidine substrate (BD OptEIA ${ }^{\mathrm{TM}}$, San Diego, CA, USA) was added to colon homogenate samples in 96-well plates at $37^{\circ} \mathrm{C}$. The reaction was stopped, and read- ings were performed in a spectrophotometer (Perkin Elmer Cetus, CA, USA) at $450 \mathrm{~nm}$. The results were normalized to the dry weight of each colon section and are expressed as the optical density per gram of tissue ( $\mathrm{nm} / \mathrm{g}$ of tissue).

For the nitric oxide measurements, the nitrite accumulated in the colon homogenates was measured as an indicator of NO production using a Griess reaction. ${ }^{20}$ The tissue homogenate was mixed 1:1 with Griess reagent (1\% sulfanilamide in $5 \%$ phosphoric acid and 0.1\% naphthyl-ethylenediamine-hydrogen chloride) and incubated at room temperature for $10 \mathrm{~min}$. The absorbance was measured at $540 \mathrm{~nm}$ in a 96-well plate reader (Perkin Elmer Cetus). The amount of nitrite in the samples was calculated based on the absorbance of a serial dilution of the sodium nitrite standard curve. The results were normalized to the dry weight of each colon section and are expressed as picograms per milliliter per gram of tissue $(\mathrm{pg} / \mathrm{mL} / \mathrm{g})$.

\section{Histopathological analysis}

To assess the microscopic damage induced by DSS in mice treated with or without SPI, the colon sections were cut longitudinally, washed with PBS, fixed in $10 \%$ buffered formalin for 24 hours, and then processed for paraffin embedding followed by microtome sectioning. Tissue sections $(5 \mu \mathrm{m})$ were obtained and stained with hematoxylin and eosin. For histopathological analysis, the mucosa, submucosa, muscle layers, and serosa were evaluated. These colon sections were also assessed for the presence of edema, inflammatory infiltrate, and epithelial abnormalities.

Images were captured using a digital video camera (Axion camicC5, Zeiss, Berlin, Germany) coupled to an optical microscope (Nikon Eclipse 50i, Nikon, Melville, NY, USA). Semi-quantitative analyses of infiltrating edema and epithelial abnormalities were also performed and classified according to the level of tissue involvement as follows: mild (>25\%), moderate $(25-50 \%)$, or severe ( $>50 \%)$. A trained pathologist who was blinded to the treatment performed the histopathological analysis.

\section{Cytokine quantification by ELISA}

Cytokines IFN- $\gamma$, TNF- $\alpha$, IL-4, IL-6, IL-10, IL-17 (BD Biosciences, San Jose, CA, USA) and IL-27 (Invitrogen eBioscience, Vienna, Austria) were quantified in the colon homogenates by ELISA according to the manufacturer's instructions. The results were normalized to the dry weight of each intestinal 
section and are expressed as nanograms per milliliter per gram of tissue $(\mathrm{ng} / \mathrm{mL} / \mathrm{g}$ of tissue).

\section{Statistics}

The number of animals per group ( $n: 6)$ was estimated based on previous publications from the authors' group using C57BL/6 mice exposed to DSS. The sample size was determined by assessing the feasibility of minimally detectable effect sizes. All results are representative of two independent experiments. The normal distribution and homogeneous variance were tested for all the variables. Parametric tests were used when the distribution was considered normal, and the variance was homogeneous: unpaired Student's $t$-test or one-way analysis of variance followed by Tukey's post hoc test. The following nonparametric tests were used in cases of non-Gaussian distribution of data: Mann-Whitney test or Kruskal-Wallis test accompanied by a Dunn's post hoc test. The results are expressed as mean $\pm S D$. The differences ob- served were considered significant when $p<0.05$. Statistical analysis was performed using GraphPad Prism, version 7.0 (GraphPad Software, La Jolla, CA, USA).

\section{RESULTS}

1. Spirulina platensis controls the colitis outcome in a dose-dependent manner

One of the most frequent clinical manifestations observed in DSS-induced inflammation is weight loss. In general, this clinical sign results from the lesions induced by DSS in the colon, which leads to the lower absorption of nutrients and weight loss. Therefore, the influence of an SPI extract on weight variations was assessed in mice exposed to DSS, as described in SUBJECTS AND METHODS. Treatment using the SPI extract resulted in reduced weight loss, particularly when administered at the highest concentration (black circle) (Fig. 1A), compared to the mice treated with the lowest concentration of SPI (50
A

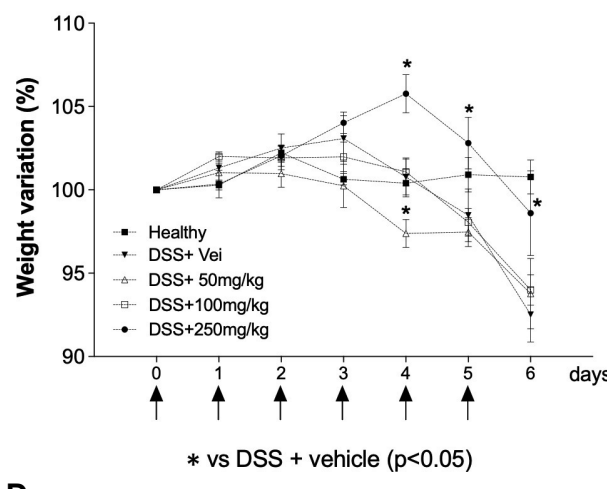

D

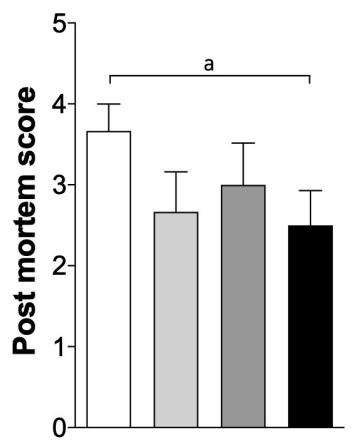

B

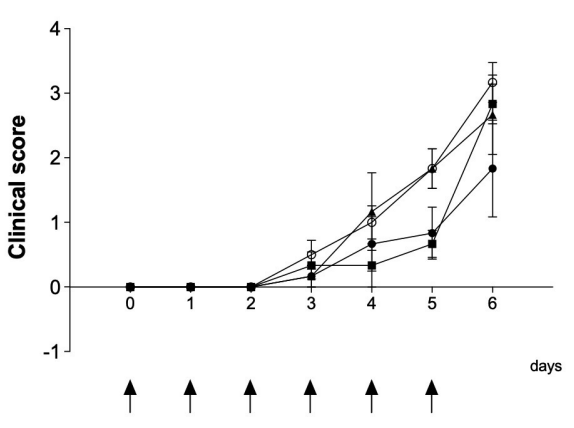

E

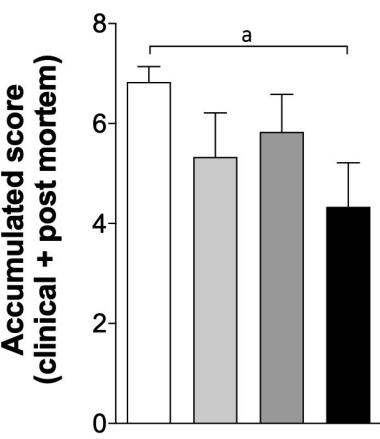

C

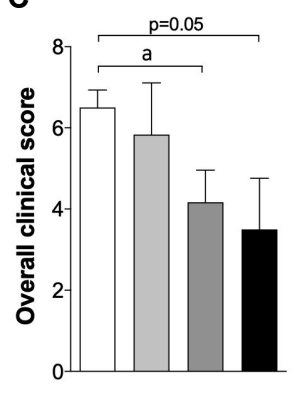

$\mathbf{F}$

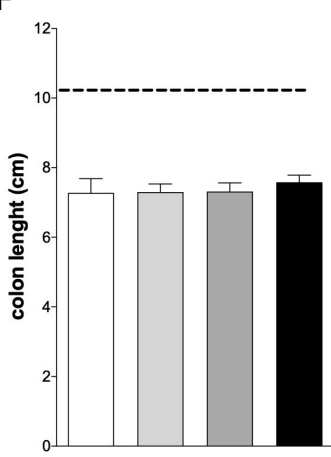

Fig. 1. SPI appears to control the colitis outcome in a dose-dependent manner. C57BL/6 male mice were exposed to DSS $3 \%$ in their drinking water for six days. The mice were treated by gavage once per day with 50,100 , or $250 \mathrm{mg} / \mathrm{kg}$ of SPI extract or saline, and euthanized on day 6. The black arrows in (A) and (B) indicate the days of treatment. Healthy: mice treated with saline only; DSS+Vei - mice exposed to DSS and treated with saline; DSS +50 or 100 or $250 \mathrm{mg} / \mathrm{kg}$ - mice exposed to DSS and treated with different concentrations of SPI. (A) weight variation; (B) clinical disease score; (C) overall clinical score; (D) post mortem score; (E) accumulated score; (F) intestinal length. The experiments were conducted with six mice/group. The results are representative of two independent experiments. DSS, dextran sulfate sodium; SPI, Arthrospira (Spirulina) platensis. ${ }^{\mathrm{a}} \mathrm{p}<0.05$. 
$\mathrm{mg} / \mathrm{kg}$ depicted as a clear triangle) or the vehicle (inverted black triangle).

The effects of SPI extract on the clinical outcome and macroscopic features of the colon were assessed. Treatment with the SPI extract reduced the disease worsening in a dose-dependent manner compared to their control counterparts (Fig. 1B, E). A significant inhibitory effect on the presentation of clinical signs of disease was observed in the mice treated with SPI at $100 \mathrm{mg} / \mathrm{kg}$ compared to the untreated mice (Fig. 1C). The beneficial effects of SPI were further evidenced by the postmortem and accumulated scores, particularly when SPI was given at $250 \mathrm{mg} / \mathrm{kg}$ (Fig. 1D, E; respectively). No difference in the colon length of mice exposed to DSS was observed, regardless of the treatment used (Fig. 1F). Overall,
SPI had a positive impact on the clinical outcomes and macroscopic features, particularly when administered at the highest concentration.

2. Spirulina platensis does not appear to influence the production of MPO and NO

After evaluating the effect of SPI extract on clinical outcomes, this study examined whether the clinical amelioration is related to the modulation of NO and MPO locally in the colon. Regardless of the treatment used, there were no significant differences in the production of MPO and NO in the tissue homogenates of mice exposed to DSS (Fig. 2A, B; respectively).
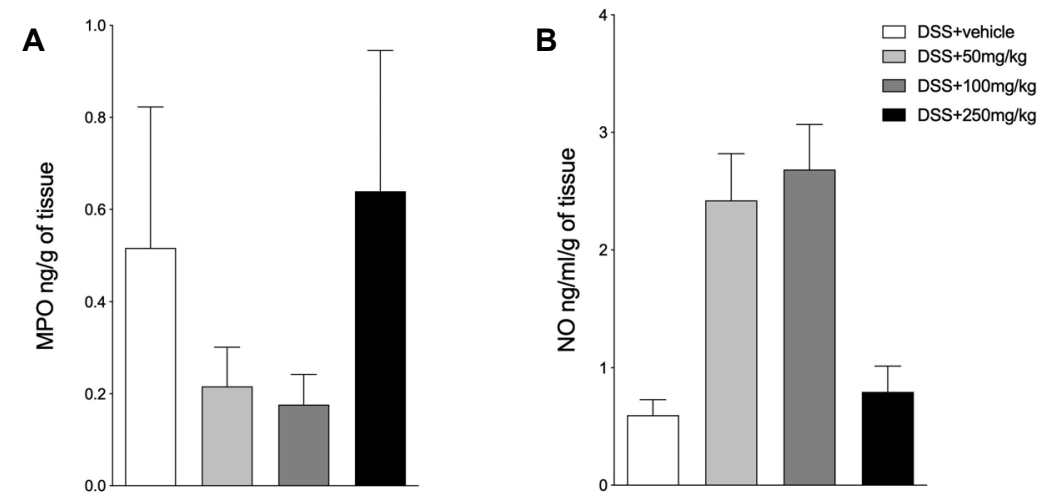

Fig. 2. SPI does not appear to affect the production MPO and NO. C57BL/6 male mice were exposed to DSS $3 \%$ in their drinking water for six days. The mice were treated by gavage with 50,100 , or $250 \mathrm{mg} / \mathrm{kg}$ of SPI extract or saline, and euthanized on day 6. (A) MPO. (B) NO. DSS+Vehicle: mice exposed to DSS and treated with saline; DSS+50 or 100 or $250 \mathrm{mg} / \mathrm{kg}$-mice exposed to DSS $3 \%$ and treated with different concentrations of SPI. The experiments were conducted with six mice/group. The results are representative of two independent experiments. MPO, myeloperoxidase; NO, nitric oxide; DSS, dextran sulfate sodium; SPI, Arthrospira (Spirulina) platensis.
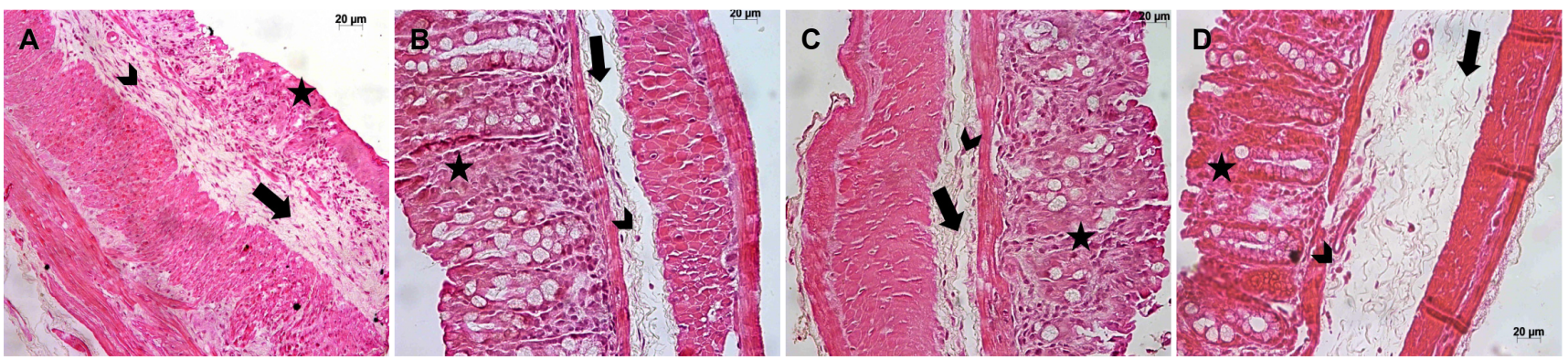

Fig. 3. Treatment with SPI attenuated DSS-induced injury in the colon. C57BL/6 male mice were exposed to DSS 3\% in the drinking water for six days. The mice were treated by gavage with 50,100 , or $250 \mathrm{mg} / \mathrm{kg}$ of SPI extract or saline, and euthanized on day 6 . In (A), the mice exposed to DSS without treatment showed moderate edema (arrow), moderate mononuclear cell infiltration (arrowhead), and intense destroyed crypts (asterisk) (H\&E, ×40). (B-D) Mice treated with A. platensis at 50, 100, or $250 \mathrm{mg} / \mathrm{kg}$, respectively, showed mild edema (arrow), mild mononuclear cell infiltration (arrowhead), and mild destroyed crypts (asterisk) (H\&E, $\times 40$ ). The experiments were conducted with six mice/group. The results are representative of two independent experiments. SPI, Arthrospira (Spirulina) platensis; DSS, dextran sulfate sodium. 
3. Treatment with Spirulina platensis attenuated DSS-induced injury in the colon

Because of the beneficial effects of SPI on the clinical outcomes, this study examined the role of SPI on the intestinal architecture. Untreated mice exposed to DSS showed moderate edema (arrow), moderate mononuclear cell infiltration (arrowhead), and intense destroyed crypts (asterisk) (Fig. 3A). In contrast, the mice treated with 50,100, and $250 \mathrm{mg} / \mathrm{kg}$ of SPI extract (Fig. 3B-D; respectively) showed mild edema (arrow), mild mononuclear cell infiltration (arrowhead), and mild destroyed crypts (asterisk). These results suggest that regardless of the concentration of SPI used, the treatment attenuated inflammation, edema, and epithelial abnormalities of the colon compared to the untreated mice exposed to DSS.

A

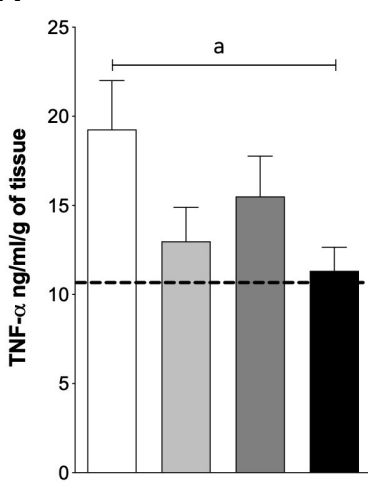

E

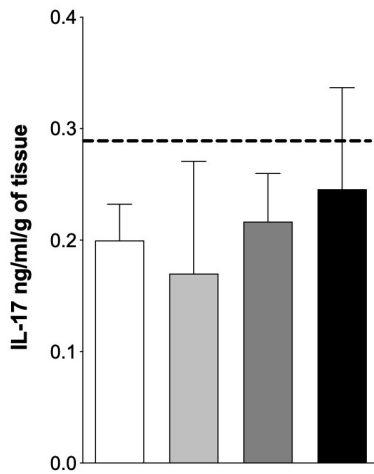

B

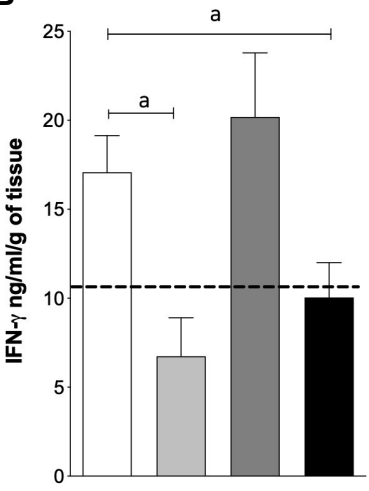

$\mathbf{F}$

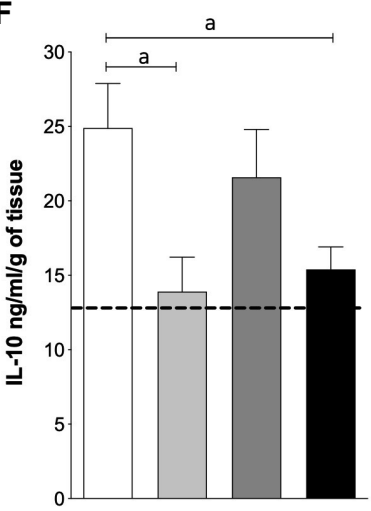

4. Spirulina platensis reduces key inflammatory cytokines to the progression of intestinal inflammation

Because the therapy using SPI improved the clinical outcomes and reduced the severity of DSS-induced injuries in the intestine, this study examined whether these ameliorations were related to the local modulation of cytokines. Mice treated with SPI, regardless of the concentration used, showed the reduced production of inflammatory cytokines, such as TNF- $\alpha$ and IFN- $\gamma$ (Fig. 4A, B; respectively). Despite the suppression of IFN- $\gamma$ production with SPI at 50 and 250 $\mathrm{mg} / \mathrm{kg}$, mice treated with SPI at $100 \mathrm{mg} / \mathrm{kg}$ produced higher levels of this cytokine, but the difference was not significant. A similar trend was observed for the quantification of TNF- $\alpha$ (Fig. 4A) and IL-10 (Fig. 4F). Surprisingly, the mice treated with SPI, particularly at 50 and $250 \mathrm{mg} / \mathrm{kg}$, showed a decrease in the production of IL-10 compared to those with intestinal inflammation and treated with vehicle only (Fig. 4F).
C
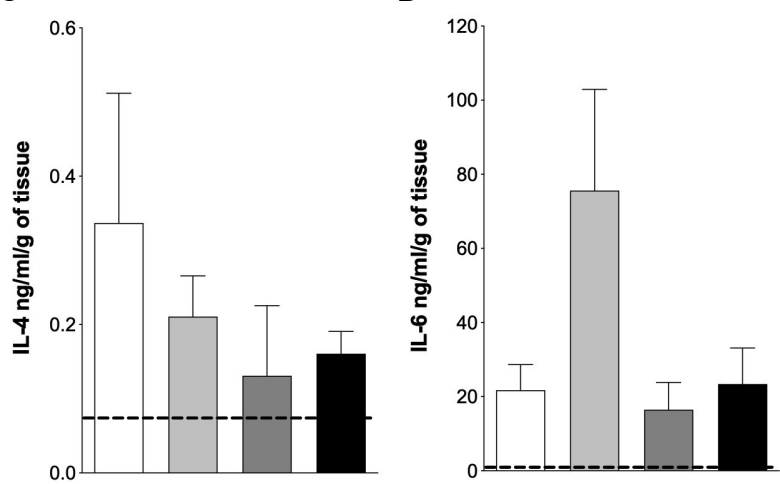

G

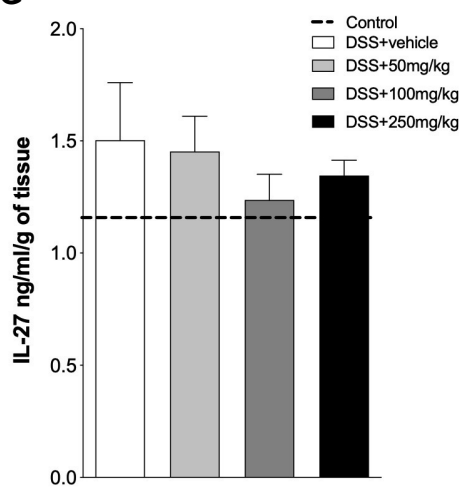

Fig. 4. SPI reduces key inflammatory cytokines to the progression of intestinal inflammation. C57BL/6 male mice were exposed to DSS $3 \%$ in their drinking water for six days. The mice were treated by gavage with 50,100 , or $250 \mathrm{mg} / \mathrm{kg}$ of SPI extract or saline, and euthanized on

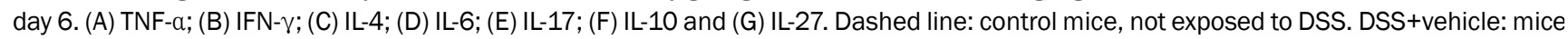
exposed to DSS and treated with saline; DSS +50 or 100 or $250 \mathrm{mg} / \mathrm{kg}$-mice exposed to DSS $3 \%$ and treated with different concentrations of SPI extract. The experiments were conducted with six mice/group. The results are representative of two independent experiments. SPI, Arthrospira (Spirulina) platensis; DSS, dextran sulfate sodium; TNF, tumor necrosis factor; IFN, interferon; IL, interleukin. ${ }^{\mathrm{a}} \mathrm{p}<0.05$. 
On the other hand, there were no differences in the production of IL-4 (Fig. 4C), IL-6 (Fig. 4D), IL-17 (Fig. 4E), and IL-27 (Fig. 4G). Overall, these results highlight the therapeutic potential of SPI extract to treat DSS-induced intestinal inflammation.

\section{DISCUSSION}

This study examined the anti-inflammatory properties of an extract of SPI in DSS-induced intestinal inflammation. At the highest dose, the extract could ameliorate the clinical outcomes and intestinal architecture as well as reduce the local production of inflammatory cytokines in the intestine, which are related to disease worsening and are used as a target for other therapeutic approaches.

The exacerbated immune response in IBD leads to the production of inflammatory cytokines, such as IFN- $\gamma$, IL-12, IL-6, TNF- $\alpha$, IL-17, and IL-23, which contribute to the development and maintenance of chronic inflammation. ${ }^{21,22}$ The current therapies are aimed at modulating the inflammatory mediators, inducing remission, and reducing disease relapse. Nevertheless, even the immune biologics targeting cytokines present several side effects and may induce intolerance.,22 This scenario highlights the need to develop therapeutic strategies with greater efficacy.

The anti-inflammatory activity of SPI appears to be related to the inhibition of $\mathrm{NO}$, prostaglandin $\mathrm{E}_{2}\left(\mathrm{PGE}_{2}\right)$, the transcription factor NF- $\mathrm{kB}$, cyclooxygenase-2 (COX-2), TNF- $\alpha$, and IL-1 $\beta$, as described previously. ${ }^{23,24}$ These properties can be at least partly attributed to phycocyanin. Phycocyanin is one of the main molecules found in spirulina that has significant antioxidant and anti-inflammatory activities. The compound can suppress the expression and production of inducible nitric oxide synthase, nitrites, as well as inhibit liver microsomal lipid peroxidation and the expression of COX-2 and $\mathrm{PGE}_{2}{ }^{23}$ Although this study did not examine if the effects observed in the present study were due to the activity of phycocyanin, the role of phycocyanin in immune modulation and clinical amelioration in DSS-induced colitis cannot be underestimated.

Spirulina has been used to re-establish the immune balance in different scenarios owing to its anti-inflammatory and immunomodulatory properties. Treatment with SPI at 200 or $400 \mathrm{mg} / \mathrm{kg}$ inhibited the development of macroscopic and microscopic lesions in Freund's adjuvant-induced arthritis in rats and improved the clinical outcomes compared to the untreated controls. ${ }^{8}$ Furthermore, SPI decreased the levels of COX-2, TNF- $\alpha$, and IL- 6 in the serum. ${ }^{8}$ Similarly, using S. maxima, the anti-inflammatory effects of Spirulina spp were reinforced in an experimental model of chronic inflammation induced by Freund's complete adjuvant in rats. ${ }^{25}$ The therapeutic and anti-inflammatory properties of SPI were also explored in experimental models of IBD, such as 2, 4, 6-TNBS, acetic acid-induced inflammation, and DSS. The oral administration of $2 \mathrm{~g} / \mathrm{kg}$ of SPI in Wistar rats 7 days after TNBS exposure induced a reduction in inflammation on the mucosa and submucosa layers, along with a decrease in necrosis, cellular infiltration, and crypt abscess formation compared to the untreated group. ${ }^{11}$ The administration of SPI at 500 $\mathrm{mg} / \mathrm{kg}$ to rats in an acetic-acid model of intestinal inflammation could improve the clinical score and reduce the production of MPO, PGE 2 , TNF- $\alpha, \mathrm{IL}-1 \beta$, and IL- 6 in the intestine. ${ }^{12}$ The oral administration of the hydroalcoholic extract of SPI at $200 \mathrm{mg} / \mathrm{kg}$ also promoted disease amelioration and reduced the DSS-related inflammatory changes, including a reduction in TNF- $\alpha$ and IL-6. ${ }^{15}$

Interestingly, mice treated with the lowest $(50 \mathrm{mg} / \mathrm{kg}$ ) or the highest $(250 \mathrm{mg} / \mathrm{kg}$ ) doses showed lower levels of the anti-inflammatory cytokine IL-10 compared to those with intestinal inflammation receiving the vehicle only. At the highest dose, this suggests that the modulation of inflammation relied more on the reduction of the key inflammatory cytokines, such as IFN- $\gamma$ and TNF- $\alpha$, than on the induction of anti-inflammatory molecules, such as IL-10. The better clinical outcome and coIon architecture observed at the highest dose corroborate this hypothesis. Despite the differences in the experimental models of inflammation, SPI concentrations, nature of the extract, and duration of treatment used in previous studies, SPI appears to be an important modulator of inflammation in intestinal inflammation. These results are in accordance with previous publications concerning the downregulation of inflammatory cytokines, particularly TNF- $\alpha$ and IFN- $\gamma$.

Furthermore, SPI could protect and reduce intestinal damage in mice challenged with DSS. Although the effects of SPI on the production of the inflammatory mediators, $\mathrm{PGE}_{2}$ and COX-2, were not evaluated, the contribution of such modulation to constrain inflammation and disease worsening cannot be excluded. In addition, treatment with SPI had no significant impact on the modulation of MPO. Intriguingly, despite 
the lack of statistical significance, the doses of 50 and 100 $\mathrm{mg} / \mathrm{kg}$ tended to induce higher levels of NO production, which indicate a dose-dependent paradoxical effect of the SPI extract. In DSS-induced inflammation in rats and using a hydroalcoholic extract, however, treatment with SPI at 100 and $200 \mathrm{mg} / \mathrm{kg}$ reduced the production of MPO. ${ }^{15}$ These differences could, at least in part, be attributed to the treatment duration and the different natures of the extracts used in the studies (saline vs. hydroalcoholic), which may have resulted in different molecular compositions.

Despite the beneficial effects observed when the SPI extract was given to the DSS-exposed mice, particularly when the highest dose was used, this study had some limitations that need to be considered when interpreting these findings. First, the molecule in the SPI extract responsible for the observed effects was not identified. Second, a dose-dependent effect was not observed in some assays, such as the determination of NO, MPO, and some cytokines. The crude extract, which is a complex formed by molecules with distinct chemical and biological characteristics and in different amounts, is not homogenous, which may have contributed to this observation. Even isolated molecules, such as $\mathrm{PGE}_{2}$, can present either pro- or anti-inflammatory properties, depending on the cell signalization, context, and dosage. ${ }^{26,27}$ This observation suggests the presence of molecules in the saline extract with putative ambiguous activity, acting as pro- or anti-inflammatory mediators depending on the dose, but such properties need to be explored further. Third, the nature of the extract, which was obtained by macerating crude SPI with saline, may also have contributed to the isolation of compounds with different chemical and biological properties.

In conclusion, these findings suggest an immunomodulatory property of SPI in modulating IFN- $\gamma$ and TNF- $\alpha$, which are the key cytokines in the development of inflammatory and autoimmune diseases, such as IBD. Furthermore, SPI, particularly at $250 \mathrm{mg} / \mathrm{kg}$, attenuated DSS-induced intestinal architecture disturbances and positively affected the clinical outcomes.

\section{REFERENCES}

1. Basso PJ, Fonseca MT, Bonfá G, et al. Association among genetic predisposition, gut microbiota, and host immune response in the etiopathogenesis of inflammatory bowel disease. Braz J Med Biol Res 2014;47:727-737.

2. Huang $Y$, Chen Z. Inflammatory bowel disease related innate immunity and adaptive immunity. Am J Transl Res 2016;8:
2490-2497.

3. Sales-Campos H, Basso PJ, Alves VB, et al. Classical and recent advances in the treatment of inflammatory bowel diseases. Braz J Med Biol Res 2015;48:96-107.

4. Kay RA. Microalgae as food and supplement. Crit Rev Food Sci Nutr 1991;30:555-573.

5. Lupatini AL, Colla LM, Canan C, Colla E. Potential application of microalga Spirulina platensis as a protein source. J Sci Food Agric 2017;97:724-732.

6. Remirez D, González R, Merino N, Rodriguez S, Ancheta O. Inhibitory effects of Spirulina in zymosan-induced arthritis in mice. Mediators Inflamm 2002;11:75-79.

7. Kumar N, Singh S, Patro N, Patro I. Evaluation of protective efficacy of Spirulina platensis against collagen-induced arthritis in rats. Inflammopharmacology 2009;17:181-190.

8. Ali EA, Barakat BM, Hassan R. Antioxidant and angiostatic effect of Spirulina platensis suspension in complete Freund's adjuvant-induced arthritis in rats. PLoS One 2015;10:e0121523.

9. Pak W, Takayama F, Mine M, et al. Anti-oxidative and anti-inflammatory effects of spirulina on rat model of non-alcoholic steatohepatitis. J Clin Biochem Nutr 2012;51:227-234.

10. Mao TK, Van de Water J, Gershwin ME. Effects of a Spirulinabased dietary supplement on cytokine production from allergic rhinitis patients. J Med Food 2005;8:27-30.

11. Coskun ZK, Kerem M, Gurbuz N, et al. The study of biochemical and histopathological effects of spirulina in rats with TNBS-induced colitis. Bratisl Lek Listy 2011;112:235-243.

12. Abdel-Daim MM, Farouk SM, Madkour FF, Azab SS. Anti-inflammatory and immunomodulatory effects of Spirulina platensis in comparison to Dunaliella salina in acetic acid-induced rat experimental colitis. Immunopharmacol Immunotoxicol 2015;37:126-139.

13. Wirtz S, Popp V, Kindermann M, et al. Chemically induced mouse models of acute and chronic intestinal inflammation. Nat Protoc 2017;12:1295-1309.

14. Wirtz S, Neufert C, Weigmann B, Neurath MF. Chemically induced mouse models of intestinal inflammation. Nat Protoc 2007;2: 541-546.

15. Morsy MA, Gupta S, Nair AB, Venugopala KN, Greish K, El-Daly M. Protective effect of Spirulina platensis extract against dextran-sulfate-sodium-induced ulcerative colitis in rats. Nutrients 2019;11:2309.

16. Perše M, Cerar A. Dextran sodium sulphate colitis mouse model: traps and tricks. J Biomed Biotechnol 2012;2012:718617.

17. Jirkof $P$, Leucht $K$, Cesarovic $N$, et al. Burrowing is a sensitive behavioural assay for monitoring general wellbeing during dextran sulfate sodium colitis in laboratory mice. Lab Anim 2013;47: 274-283.

18. Sales-Campos H, de Souza PR, Basso PJ, et al. Aedes aegypti salivary gland extract ameliorates experimental inflammatory bowel disease. Int Immunopharmacol 2015;26:13-22.

19. Coutinho de Sousa B, Reis Machado J, da Silva MV, et al. Morinda citrifolia (Noni) fruit juice reduces inflammatory cytokines expression and contributes to the maintenance of intestinal mucosal integrity in DSS experimental colitis. Mediators Inflamm 2017;2017:6567432. 
20. Green LC, Ruiz de Luzuriaga K, Wagner DA, et al. Nitrate biosynthesis in man. Proc Natl Acad Sci U S A 1981;78:7764-7768.

21. Fonseca-Camarillo G, Yamamoto-Furusho JK. Immunoregulatory pathways involved in inflammatory bowel disease. Inflamm Bowel Dis 2015;21:2188-2193.

22. Yamamoto-Furusho JK. Inflammatory bowel disease therapy: blockade of cytokines and cytokine signaling pathways. Curr Opin Gastroenterol 2018;34:187-193.

23. Deng R, Chow TJ. Hypolipidemic, antioxidant, and antiinflammatory activities of microalgae Spirulina. Cardiovasc Ther 2010;28:e33-e45.

24. Ku CS, Pham TX, Park Y, et al. Edible blue-green algae reduce the production of pro-inflammatory cytokines by inhibiting NF-KB pathway in macrophages and splenocytes. Biochim Biophys Acta 2013;1830:2981-2988.

25. Gutiérrez-Rebolledo GA, Galar-Martínez M, García-Rodríguez RV, Chamorro-Cevallos GA, Hernández-Reyes AG, Martínez-Galero E. Antioxidant effect of Spirulina (arthrospira) maxima on chronic inflammation induced by Freund's complete adjuvant in rats. J Med Food 2015;18:865-871.

26. Ricciotti E, FitzGerald GA. Prostaglandins and inflammation. Arterioscler Thromb Vasc Biol 2011;31:986-1000.

27. Osma-Garcia IC, Punzón C, Fresno M, Díaz-Muñoz MD. Dose-dependent effects of prostaglandin E2 in macrophage adhesion and migration. Eur J Immunol 2016;46:677-688. 